\title{
MR Imaging of Individual Perfusion Reorganization Using Superselective Pseudocontinuous Arterial Spin-Labeling in Patients with Complex Extracranial Steno-Occlusive Disease
}

\author{
(D) V. Richter, (D) M. Helle, (DM.J.P. van Osch, (D). Lindner, (D)A.S. Gersing, DP. Tsantilas, (DH.-H. Eckstein, (DC. Preibisch, and
} (D) C. Zimmer

\begin{abstract}
BACKGROUND AND PURPOSE: Patients with multiple stenoses or occlusions of the extracranial arteries require an individualized diagnostic approach. We evaluated the feasibility and clinical utility of a novel MR imaging technique for regional perfusion imaging in this patient group.
\end{abstract}

MATERIALS AND METHODS: Superselective pseudocontinuous arterial spin-labeling with a circular labeling spot enabling selective vessel labeling was added to routine imaging in a prospective pilot study in 50 patients (10 women, $70.05 \pm 10.55$ years of age) with extracranial steno-occlusive disease. Thirty-three had infarct lesions. DSC-MR imaging was performed in 16/50 (32\%), and cerebral DSA, in 12/50 patients (24\%). Vascular anatomy and the distribution of vessel stenoses and occlusions were defined on sonography and TOF-MRA. Stenoses were classified according to the NASCET criteria. Infarct lesions and perfusion deficits were defined on FLAIR and DSC-MR imaging, respectively. Individual perfusion patterns were defined on the superselective pseudocontinuous arterial spin-labeling maps and were correlated with vascular anatomy and infarct lesion localization.

RESULTS: The superselective pseudocontinuous arterial spin-labeling imaging sequence could be readily applied by trained technicians, and the additional scan time of 12.7 minutes was well-tolerated by patients. The detected vessel occlusions/stenoses and perfusion patterns corresponded between cerebral DSA and superselective pseudocontinuous arterial spin-labeling maps in all cases. Perfusion deficits on DSC-CBF maps significantly correlated with those on superselective pseudocontinuous arterial spin-labeling maps (Pearson $r=$ $0.9593, P<.01)$. Individual collateral recruitment patterns were not predictable from the vascular anatomy in $71 \%$ of our patients.

CONCLUSIONS: Superselective pseudocontinuous arterial spin-labeling is a robust technique for regional brain perfusion imaging, suitable for the noninvasive diagnostics of individual perfusion patterns in patients with complex cerebrovascular disease.

ABBREVIATIONS: AcomA = anterior communicating artery; $\mathrm{ASL}=$ arterial spin-labeling; $\mathrm{CDSA}=$ cerebral DSA; $\mathrm{ECA}=$ external carotid artery; $\mathrm{pCASL}=\mathrm{pseudo-}$ continuous arterial spin-labeling; PcomA = posterior communicating artery; ss-pCASL= superselective pseudocontinuous arterial spin-labeling; VA = vertebral artery

n patients with complex steno-occlusive disease of the extracranial brain-supplying arteries, it is often difficult to determine the culprit source of an ischemic lesion. CT and MR angiography provide morphologic information on the configuration of the circle of Willis. Clinically accepted methods to visualize brain perfu-

Received August 21, 2016; accepted after revision December 4.

From the Department of Radiology (V.R.), Diagnostic and Interventional Radiology, University of Tübingen, Tübingen, Germany; Department of Radiology and Neuroradiology (M.H., T.L.), University Medical Center Schleswig-Holstein, Kiel, Germany Philips GmbH Innovative Technologies (M.H.), Research Laboratories, Hamburg, Germany; The C. J. Gorter Center for High Field MRI (M.J.P.v.O.), Department of Radiology, Leiden University Medical Center, Leiden, The Netherlands; and Departments of Diagnostic and Interventional Neuroradiology (A.S.G., C.P., C.Z.) and Vascular and Endovascular Surgery (P.T., H.-H.E.), Klinikum Rechts der Isar, Technical University Munich, Munich, Germany.

Please address correspondence to Vivien Richter, MD, Department of Radiology, Diagnostic and Interventional Radiology, University of Tübingen, Hoppe-Seyler-Str 3, 72076 Tübingen, Germany; e-mail: vivien.richter@med.uni-tuebingen.de

$\equiv$ Indicates article with supplemental on-line table.

http://dx.doi.org/10.3174/ajnr.A5090 sion such as $\mathrm{CT}$ and $\mathrm{MR}$ imaging ${ }^{1}$ usually require contrast agent injection and do not allow the assessment of collateral flow. However, collateral pathways, including the circle of Willis and leptomeningeal collaterals, are recruited in a highly individual pattern to compensate for diminished blood flow, as demonstrated by studies showing a wide variability in perfusion territories of brain arteries in healthy individuals ${ }^{2-6}$ and even more so in those with steno-occlusive disease. ${ }^{7-13}$ Imaging of individual perfusion territories allows the visualization of this variability in individual patients and thus permits conclusions concerning collateral flow and subsequently reorganized perfusion patterns. ${ }^{10-14}$ It may pinpoint the source of the greatest hemodynamic impairment and-by visualizing the localization of the infarct in relation to the individual perfusion patterns-may aid in differentiating thromboembolic stroke and ischemic lesions due to compromised cerebral perfusion in watershed areas. This information is essential for the prevention of recurrent stroke. ${ }^{15}$

The criterion standard of vessel-selective imaging is digital 
subtraction angiography, which enables imaging of macrovascular blood flow and reorganization of perfusion territories, allowing only indirect assumptions about microvascular perfusion. Its invasiveness and the associated risks ${ }^{16}$ as well as concerns about radiation exposure advocate for the development of noninvasive alternatives. The criterion standard of cerebral perfusion imaging is positron-emission tomography, ${ }^{17}$ which provides information about microvascular perfusion without correlation to macrovascular blood flow, but again it is an invasive technique associated with radiation exposure.

MR imaging-based methods for perfusion imaging with vessel-selective arterial spin-labeling (ASL) have been developed to provide a tool for noninvasive regional perfusion imaging. ${ }^{18-20}$ The first reports on territorial arterial spin-labeling were presented in 2003, ${ }^{21}$ and techniques for vessel-selective labeling have since been improved with respect to vessel selectivity, ${ }^{22}$ usability, ${ }^{23}$ and data analysis. ${ }^{24}$ Initial techniques for vessel-selective labeling required positioning a thick labeling slab in a plane with the aim of selectively labeling only 1 artery. ${ }^{12}$ More recently, in a practically planning-free approach, the vessel-encoded pseudocontinuous arterial spin-labeling (pCASL) ${ }^{25}$ technique was proposed, which uses phase cycling and additional gradients to achieve a spatially varying labeling efficiency within the labeling plane. This allows simultaneous acquisition of several perfusion territories in $<5$ minutes. However, the required evaluation procedure is highly sophisticated and uses comparison with a full perfusion map by iterative clustering methods. ${ }^{19}$

The variability of labeling approaches and evaluation procedures is manifold and standardized recommendations-such as those for conventional $\mathrm{ASL}^{26}$ — are not yet available. These features, along with lacking product sequences and established postprocessing procedures, limit the clinical use of vessel-selective ASL, despite promising results. ${ }^{27-31}$

The superselective pseudocontinuous ASL (ss-pCASL) technique with a single circular labeling spot ${ }^{22,32}$ works by applying rotating gradients with phase changes of the radiofrequency pulses of the pCASL labeling train, in which the diameter of the labeling focus is controlled by the gradient moments. ${ }^{22,32}$ The major advantage of superselective pCASL is its flexibility in positioning: The labeling focus can be individually adapted to different vessel geometries (eg, by tilting). This adaptation can be especially beneficial in patients with altered vasculatures such as tortuous and elongated vessels. Planning the ss-pCASL is similar to that in conventional pCASL; however, instead of a labeling plane, a labeling focus needs to be located over the artery of interest, which usually only takes a short amount of additional time for trained MR imaging operators. Another advantage is the straightforward evaluation procedure: Relative perfusion maps are created on the basis of simple correlation procedures and can be easily visualized in real-time at the scanner console. To our knowledge, no clinical case series has yet been examined with this technique.

The aim of our study was 2-fold: 1) to determine the feasibility of superselective pCASL in everyday clinical practice with regard to scanning time, technician training, and patient tolerability; and 2) to demonstrate its accuracy by correlating the imaging of flow territories with DSC-PWI or cerebral DSA (cDSA). In addition, we demonstrate the high individual variability of perfusion patterns in a patient population with complex chronic steno-occlusive disease of the extracranial brain-supplying arteries.

In summary, we evaluated the feasibility and clinical utility of a novel MR imaging technique for regional perfusion imaging in this patient group.

\section{MATERIALS AND METHODS \\ Patients}

Fifty consecutive patients ( 10 women; mean age, $70.05 \pm 10.55$ years) with stenosis and/or occlusion of $\geq 1$ extracranial artery were examined with ss-pCASL, in addition to clinical routine MR imaging, between February 2012 and August 2014 in a prospective pilot study. Patients underwent neurologic work-ups due to previous transient ischemic attacks or a nondisabling ischemic stroke. Steno-occlusive disease had been diagnosed before referral with duplex ultrasonography. All patients included in our study had a sonographically detected high-grade internal carotid artery and/or vertebral artery (VA) stenosis $(>70 \%$ according to the North American Symptomatic Carotid Endarterectomy [NASCET] grading) or a vessel occlusion. Thirtythree of 50 (66\%) patients had subacute (older than 1 week) or chronic (older than 1 month) infarct lesions. After we excluded MR imaging contraindications, all patients gave written informed consent. Sixteen of $50(32 \%)$ patients underwent contrast-enhanced MR perfusion imaging (DSC-PWI), and 12/50 patients (24\%) underwent cerebral DSA within 2 weeks of the ss-pCASL study. Two of 50 patients were excluded due to motion artifacts on the MR imaging.

\section{MR Imaging}

All planning steps were performed by a neuroradiology technician after an initial 30-minute training session about loading, planning, and running the ss-pCASL sequence before actual clinical imaging. MR imaging was performed on a 3T whole-body system (Achieva; Philips Healthcare, Best, the Netherlands) with the whole-body radiofrequency coil for transmission and a 16channel phased array head and neck coil for signal reception. After a sensitivity encoding reference scan, a fast time-of-flight angiography was performed with MIPs to display vascular anatomy ranging from the carotid bifurcation to the skull base (scan parameters: FOV, $230 \times 230 \times 81 \mathrm{~mm}^{3}$; acquisition voxel size, $1.19 \times 2.39 \times 3 \mathrm{~mm}^{3}$ [reconstructed, $0.89 \times 0.89 \times 3 \mathrm{~mm}^{3}$ ]; flip angle, $60^{\circ}$; $3 \mathrm{D}$ fast-field echo; TR/TE, $25 / 3.5 \mathrm{~ms}$; acquisition time, 1 minute 40 seconds). This sequence was used for planning of the labeling planes.

Anatomic sequences included FLAIR (FOV, $230 \times 230 \times 144$ $\mathrm{mm}^{3}$; acquisition voxel size, $0.90 \times 0.97 \times 4 \mathrm{~mm}^{3}$ [reconstructed, $0.45 \times 0.45 \times 4 \mathrm{~mm}^{3}$ ]; flip angle, $90^{\circ}$; TSE factor, 38 ; TR/TE, 12,000/140 ms; inversion delay, $2850 \mathrm{~ms}$; acquisition time, $3 \mathrm{~min}$ utes); DWI (spin-echo EPI; FOV, $224 \times 224 \times 146 \mathrm{~mm}^{3}$; acquisition voxel size, $2 \times 2 \times 2 \mathrm{~mm}^{3}$ [reconstructed, $0.875 \times 0.875 \times$ $2 \mathrm{~mm}^{3}$ ]; flip angle, $90^{\circ}$; sensitivity encoding factor, 2 ; TR/TE, 7845/55 ms; b-factor, 1000; acquisition time, 2 minutes $37 \mathrm{sec}$ onds); T2WI fast-field echo (FOV, $230 \times 230 \times 144 \mathrm{~mm}^{3}$; acquisition voxel size, $0.89 \times 1.12 \times 4 \mathrm{~mm}^{3}$ [reconstructed, $0.45 \times$ $0.45 \times 4 \mathrm{~mm}^{3}$ ]; flip angle, $18^{\circ}$; TR/TE, 956/16 ms; acquisition 


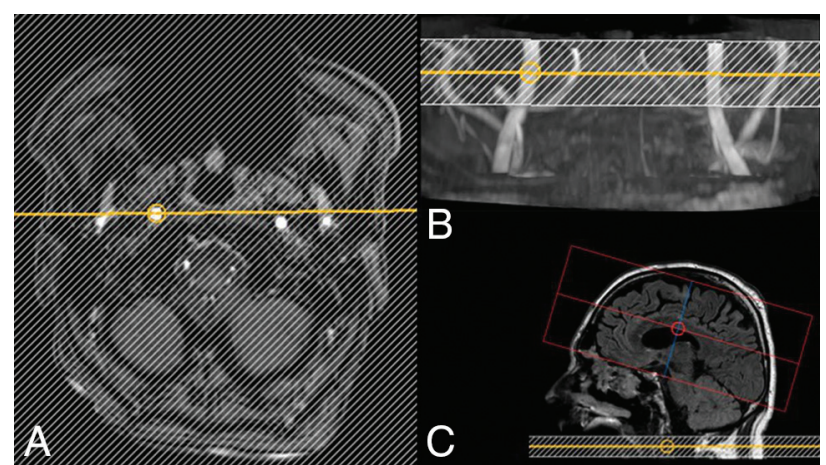

FIG 1. Planning steps of the superselective PCASL sequence. The labeling stack is positioned on a fast-TOF-MRA of the cervical vessels in the axial plane $(A)$ and on the MIP projection $(B)$. C, The readout slab is positioned covering the expected brain perfusion territory.

time, 2 minutes 37 seconds); and an intracranial TOF angiography (3D-fast-field echo; FOV, $200 \times 200 \times 84 \mathrm{~mm}^{3}$; acquisition voxel size, $0.40 \times 0.70 \times 0.60 \mathrm{~mm}^{3}$ [reconstructed, $0.39 \times 0.39 \times$ $0.60 \mathrm{~mm}^{3}$ ]; 4 slabs; flip angle, $20^{\circ}$; TR/TE, $25 / 3.5 \mathrm{~ms}$; sensitivity encoding factor, 2; acquisition time, 6 minutes).

During these scans, labeling positions of the ss-pCASL sequences were planned on the fast TOF scans: The circular labeling spot was positioned in the graphic user interface to cover the target vessel in its craniocaudal extension. The diameter of the circular label focus was set to $15 \mathrm{~mm}$. The labeling plane was positioned in the same angulation as the fast TOF (Fig 1). If patient movement was detected during the examination, the TOF scan was repeated and labeling locations were updated directly before performing the ss-pCASL sequence to minimize loss of labeling efficiency due to motion.

The readout module of the ss-pCASL sequence was a singleshot echo-planar imaging acquisition with the following parameters: flip angle, $90^{\circ}$; TE/TR, 12/3550 ms; FOV, $220 \times 220 \times 98.4$ $\mathrm{mm}^{3}$; voxel size, $2.7 \times 2.7 \times 6 \mathrm{~mm}^{3}$. The labeling duration was $1650 \mathrm{~ms}$, with a postlabeling delay of $1525 \mathrm{~ms}$ and background suppression pulses at 1680 and $2830 \mathrm{~ms}$ after a saturation pulse preceding the labeling. ${ }^{22,32}$ Intracranial perfusion measurements comprised 15 sections covering most perfused brain tissue. Forty averages of label and control images were acquired with an average scan time of 2 minutes 25 seconds per vessel. As a control for the detection of a potential misplacement of labeling spots and for comparison with the DSC-based CBF maps, nonselective perfusion measurements were also performed with the same parameters but without applying the selection gradients (unlike vessel-encoded pCASL, superselective pCASL does not allow reconstruction of nonselective maps). In general, the ICA and VA were labeled; the external carotid artery (ECA) was only selectively labeled in cases with bilateral ICA occlusion.

Finally, DSC perfusion data were acquired during injection of $15 \mathrm{~mL}$ of Gd-DTPA by using single-shot EPI (flip angle, $75^{\circ}$; TE/ TR, 40/1573 ms; FOV, $224 \times 224 \times 110 \mathrm{~mm}^{3}$; acquisition voxel size, $2.33 \times 2.33 \times 4 \mathrm{~mm}^{3}$ [reconstructed, $1.75 \times 1.75 \times 4 \mathrm{~mm}^{3}$ ]; 40 dynamics).

\section{Postprocessing}

The acquired ss-pCASL data were postprocessed on the scanner console by using the standard iViewBOLD package (Philips
Healthcare). A predefined protocol was applied to each measurement to generate a correlation of the labeled and nonlabeled ASL acquisitions according to an on-off paradigm; no manual thresholding or postprocessing was required apart from creating these pCASL maps, which corresponded to qualitative CBF maps. For anatomic reference, the individual flow-territory maps were overlaid on FLAIR images. These color overlay images were then transferred to the PACS for diagnostic use.

DSC-PWI CBF maps were created by using the NeuroPerfusion analysis tool (Philips Healthcare) on the Extended MR Workspace (Philips Healthcare). The analysis was based on a voxelwise deconvolution between tissue time courses and an arterial input function. The standard evaluation protocol uses weak spatial and temporal smoothing and requires the manual selection of 3-5 arterial input function voxels, which is aided by a display of temporal curves in a preselected ROI around arterial vessels from which arterial input function-like curves showing early, steep and narrow peaks are selected.

\section{Data Analysis}

Infarct lesions were identified on the FLAIR images by drawing a $3 \mathrm{D}-\mathrm{VOI}$ by using the semiautomatic volume definition tool in Brainlab (Brainlab, Munich, Germany) by 2 experienced neuroradiologists in consensus (V.R., 4 years of experience, and A.S.G. 3 years of experience).

Vascular anatomy (occlusions, stenoses, circle of Willis anatomy) was defined on the TOF-MIP images (by V.R. and A.S.G.).

cDSA examinations $(n=12$, bilateral common carotid artery, ICA, and VA injections unless catheterization was not possible) were evaluated in the clinical routine with classification of vessel occlusions and stenoses according to the NASCET criteria. The sonographically diagnosed occlusions or $>70 \%$ extracranial ICA/VA stenoses or both were confirmed by cDSA in all patients. Asymmetric perfusion patterns and collateral recruitment in cDSA were determined by using a standardized angiographic anatomy atlas. ${ }^{33}$ Vessel-by-vessel correlation of cDSA with sspCASL was performed by V.R. and A.S.G. (both blinded to clinical information): The angiographically detected vessel occlusions were correlated with a completely absent perfusion signal in the ss-pCASL and stenoses, with relevantly decreased sizes of vessel perfusion territories compared with standardized perfusion territory atlases. ${ }^{34,35}$

For correlation with the DSC perfusion method, VOIs of the CBF lesions were created on the DSC and the nonselective pCASL CBF maps, with manual definition of the areas via the visual detection of decreases in CBF (performed by V.R. and A.S.G. in consensus) compared with surrounding and contralateral brain tissue (similar to clinical routine). A 3D lesion volume was created by the semiautomatic volume definition tool in Brainlab with calculation of lesion volume in milliliters. A correlation analysis was performed by comparing these volumes visually and by pairwise calculation of the Dice similarity coefficient to quantify the degree of overlap according to the following equation ${ }^{36}$ :

Dice Coefficient $=$

$2 \times$ Volume Overlap(DSC Lesion $\cap$ pcASL Lesion) Volume DSC Lesion + Volume pcASL Lesion

AJNR Am J Neuroradiol 38:703-11 Apr 2017 www.ajnr.org 


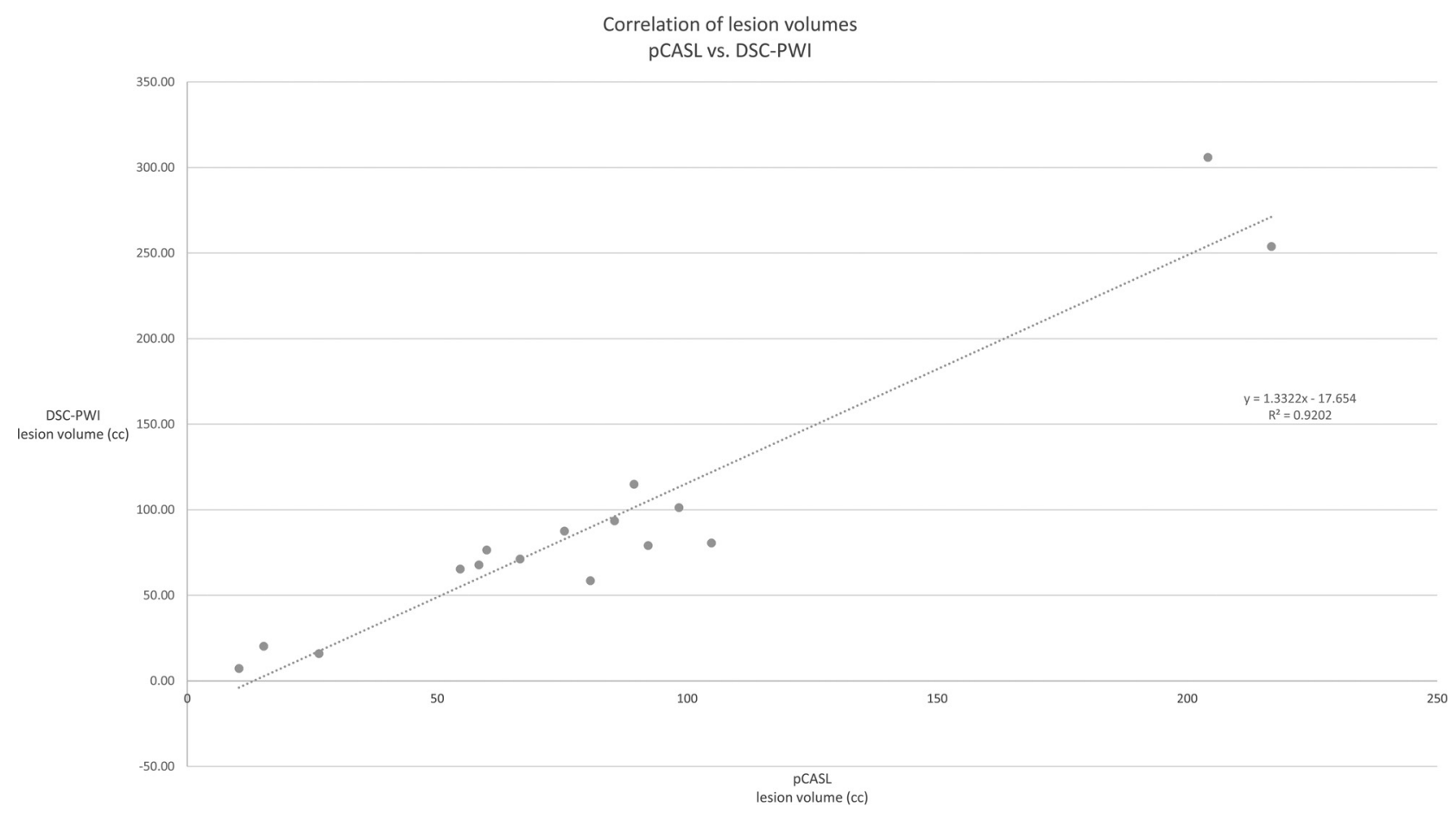

FIG 2. Correlation between PCASL- (global labeling) and DSC-PWI-identified infarct lesion volumes.

All statistical analyses were performed with GraphPad Prism software (Prism V.5 for Mac Os X; GraphPad Software, San Diego, California).

Patients were grouped into 4 categories (On-line Table) according to the presence of stenoses and occlusion: 1) unilateral high-grade ICA stenosis, 2) bilateral high-grade ICA stenosis, 3) unilateral ICA occlusion and contralateral high-grade ICA stenosis, and 4) bilateral ICA occlusion.

Perfusion territories were defined by comparing the ss-pCASL maps with a standardized perfusion territory atlas. ${ }^{34,35}$ Individual perfusion patterns were classified as follows: 1) normal pattern, 2) perfusion from contralateral ICA, 3) perfusion from the contralateral ICA and posterior circulation, and 4) perfusion only from the posterior circulation. Predictability was defined according to the following decision algorithm: 1) If either the anterior communicating artery (AcomA) or both posterior communicating arteries (PcomAs) were atretic, the new perfusion pattern was defined as "predictable" because there is only 1 remaining possibility for collateralization through the circle of Willis (eg, in the case of a unilateral ICA stenosis with a hypoplastic AcomA and both PcomAs patent, collateralization from the posterior circulation can be predicted from the vascular anatomy without regional perfusion imaging); and 2) in all other cases with combinations of patent AcomA and PcomA vessels, the new perfusion pattern was defined as "not predictable" because there is $>1$ possible way for collateralization through the circle of Willis.

The localization of infarct lesions was defined as follows: 1) relative to standardized perfusion territory atlases ${ }^{34,35}$; and 2) relative to the perfusion territories as defined by the ss-pCASL maps. An infarct lesion was considered "territorial" if $>60 \%$ of its volume overlapped a particular perfusion territory and as "watershed" if 2 adjacent perfusion territories overlapped $40 \%-60 \%$ of the lesion volume, respectively. The infarct categorization accord- ing to the standardized atlas versus the ss-pcASL method was compared for all patients.

\section{RESULTS}

\section{Feasibility and Accordance with CDSA and DSC-PWI}

Technical performance of the superselective pCASL sequence was robust, meaning that technicians were able to reliably perform the measurement to yield territorial perfusion maps of diagnostic quality. Each technician received a 30 -minute training session about loading, planning, and running the ss-pCASL sequence before actual clinical imaging. All of them performed the following measurements without assistance, and no examination had to be discarded because of flaws in planning. Postprocessing was performed on the scanner console with immediate transfer of the resulting flow territory maps to the PACS. Two of 50 patients had to be excluded due to motion artifacts. A mean of $3.48 \pm 0.79$ vessels were labeled per examination (hypoplastic or occluded vessels were not labeled). Additional scan time due to the nonselective and selective pCASL sequences was 12.7 minutes on average, depending on the number of vessels (2-5) labeled. Overall imaging time was 26 minutes (range, 24-31 minutes).

Correlation of pCASL and cDSA scores showed $100 \%$ correspondence for the detection of vessel occlusions/stenoses and for the presence of a nonsymmetric perfusion pattern, with an asymmetric perfusion pattern in $8 / 12$ patients between both readers (therefore, we refrained from calculating an interrater variability).

Mean CBF lesion volumes defined on the DSC (93.67 \pm $\left.105.67 \mathrm{~cm}^{3}\right)$ and the nonselective pCASL maps $(82.43 \pm 98.42$ $\left.\mathrm{cm}^{3}\right)$ correlated significantly $(n=16$, Pearson $r=0.9593, P<$ .01 ) (Fig 2). The degree of overlap (Dice coefficient) was $0.98 \pm$ 0.7 . 
No. of subacute or chronic infarcts in the patient subgroups (defined on FLAIR imaging), their categorization as watershed or territorial infarcts, and the number of cases in which an infarct lesion was categorized differently after ss-pCASL imaging than with the standardized perfusion atlas

\begin{tabular}{|c|c|c|c|c|}
\hline Vessel Status & $\begin{array}{c}\text { No. of Infarct } \\
\text { Lesions/No. of } \\
\text { Patients in Subgroup }\end{array}$ & $\begin{array}{l}\text { No. of Watershed } \\
\text { Infarcts/No. of } \\
\text { Infarct Lesions } \\
\text { in Subgroup }\end{array}$ & $\begin{array}{l}\text { No. of Territorial } \\
\text { Infarcts/No. of } \\
\text { Infarct Lesions } \\
\text { in Subgroup }\end{array}$ & $\begin{array}{l}\text { No. of Infarct } \\
\text { Lesions Differently } \\
\text { Categorized by Using } \\
\text { the Standardized } \\
\text { Perfusion Atlas } \\
\text { vs ss-pCASL }\end{array}$ \\
\hline Unilateral ICA stenosis & $11 / 21(52 \%)$ & $5 / 11(45 \%)$ & $6 / 11(55 \%)$ & $4 / 11(36 \%)$ \\
\hline Bilateral ICA stenosis & $4 / 9(44 \%)$ & $2 / 4(50 \%)$ & $2 / 4(50 \%)$ & $1 / 4(25 \%)$ \\
\hline $\begin{array}{r}\text { Unilateral ICA occlusion and } \\
\text { contralateral ICA stenosis }\end{array}$ & 15/15 (100\%) & $8 / 15(54 \%)$ & $7 / 15(46 \%)$ & $6 / 15(40 \%)$ \\
\hline Bilateral ICA occlusion & $3 / 3(100 \%)$ & $3 / 3(100 \%)$ & - & $0 / 3(0 \%)$ \\
\hline Sum & $33 / 48(69 \%)$ & $18 / 33(55 \%)$ & $15 / 33(45 \%)$ & $11 / 33(33 \%)$ \\
\hline
\end{tabular}

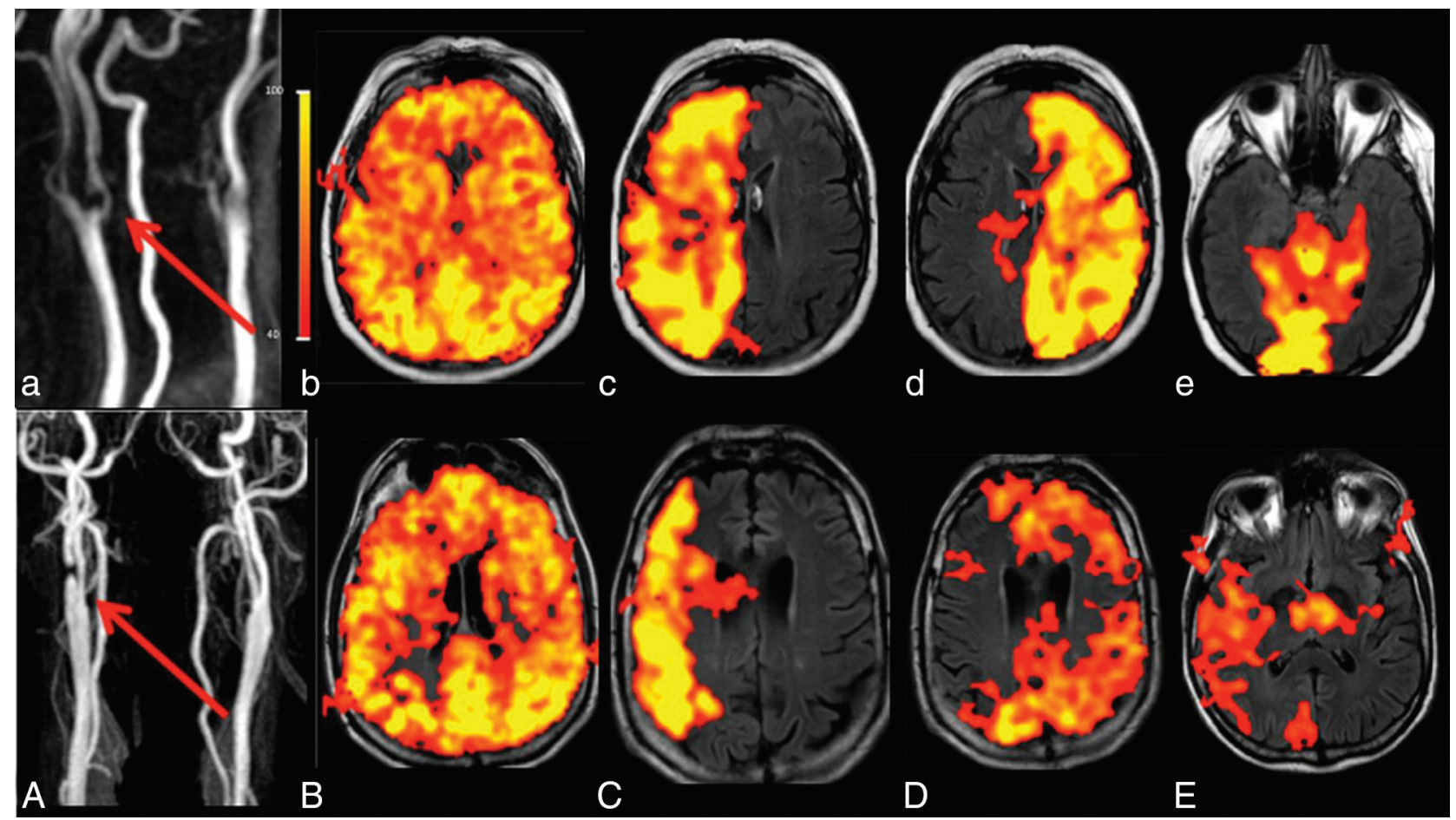

FIG 3. Illustration of individual perfusion patterns in 2 different patients with similar chronic high-grade $(>70 \%)$ right bifurcation ICA stenosis. The red arrows indicate the stenosis in patient $1(a)$ and patient $2(A)$. Both patients had patent AcomAs and bilateral PcomAs. Nonselective labeling in $b$ and $B$ shows no significant perfusion deficits. These patients did not have any infarct lesions. Labeling was performed for the right ICA, left ICA, and right VA in both patients (the left VA was hypoplastic in both cases). In patient 1 (upper row), the stenotic right ICA (c) continues to provide perfusion to the right cerebral hemisphere; The left ICA (d) and right VA (e) do not collateralize. In patient 2 (bottom row), right ICA perfusion is diminished $(C)$ and supplies only the MCA territory; the anterior and posterior territories are perfused by recruitment of the left ICA $(D)$ and right VA $(E)$. These cases illustrate situations in which the individual perfusion pattern is not predictable: Even though extracranial stenoses and circle of Willis anatomies are similar, the perfusion patterns are different.

\section{Individual Variability of Perfusion Territories}

Twenty-one patients had a unilateral ICA stenosis, while 9 patients had bilateral ICA stenoses. Fifteen patients had a unilateral ICA occlusion with a contralateral ICA stenosis, and 3 patients had bilateral ICA occlusions. The individual perfusion territories and the anatomic variants of the circle of Willis are detailed in the On-line Table. Perfusion patterns were assessed as predictable from the circle of Willis anatomy in 14/48 (29\%) patients and as not predictable in $34 / 48$ (71\%) patients.

The localization of the infarct lesions (watershed or territorial) in the subgroups of patients with different combinations of stenoses and occlusions is shown in the Table. We found that in $33 \%$ of our patients, ss-pcASL led to a different infarct categorization than the standardized atlas method.

Representative patient studies are shown in Figs 3 and 4.

In 1 case (Fig 5), ss-pCASL was performed postoperatively after carotid endarterectomy (alas, no preoperative pCASL was performed). In postoperative follow-up, a normalized territorial distribution was shown with a symmetric and comprehensive perfusion signal after labeling both ICAs. ${ }^{10}$

\section{DISCUSSION}

In this study, we demonstrate the feasibility of ss-pCASL in a clinical context. The labeling of individual arteries with a circular 


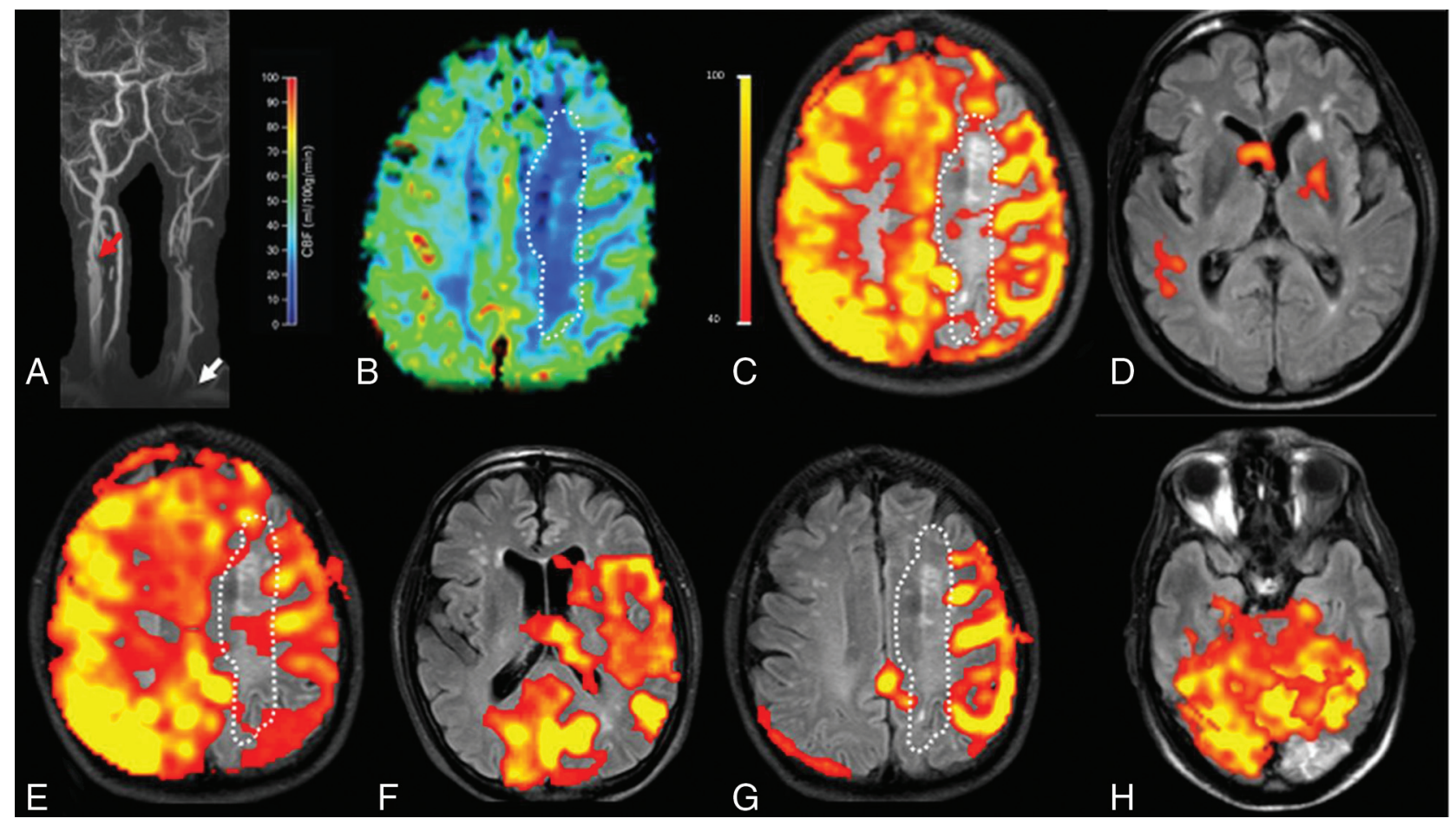

FIG 4. A 78-year-old male patient with extracranial left ICA occlusion and an extracranial high-grade right ICA stenosis (red arrow) and a left VA stenosis in the $\mathrm{V} 1$ segment (white arrow, A). The AcomA and both PcomAs are patent. A perfusion deficit (white dotted line) is seen in the left corona radiata in both the DSC-PWI $(B)$ and the nonselective PCASL map (C). Selective labeling was performed for the left common carotid artery $(D)$, right ICA $(E)$, right $\mathrm{VA}(F$ and $G)$, and left $\mathrm{VA}(H)$. The intracranial perfusion signal is missing on labeling of the left common carotid artery $(D)$ as a proof of left ICA occlusion. Right ICA labeling $(E)$ shows perfusion of the left anterior cerebral artery and MCA territory. Right VA labeling $(F$ and $G)$ shows recruitment of the posterior circulation for the perfusion of the left MCA territory. The new watershed region with a perfusion deficit and chronic infarcts is seen between the posterior circulation and the right ICA ( $E$ and $G$ ). A subacute infarct lesion in the left occipital lobe is localized within the perfusion territory of the left VA $(H)$ and can be categorized as a territorial infarct.

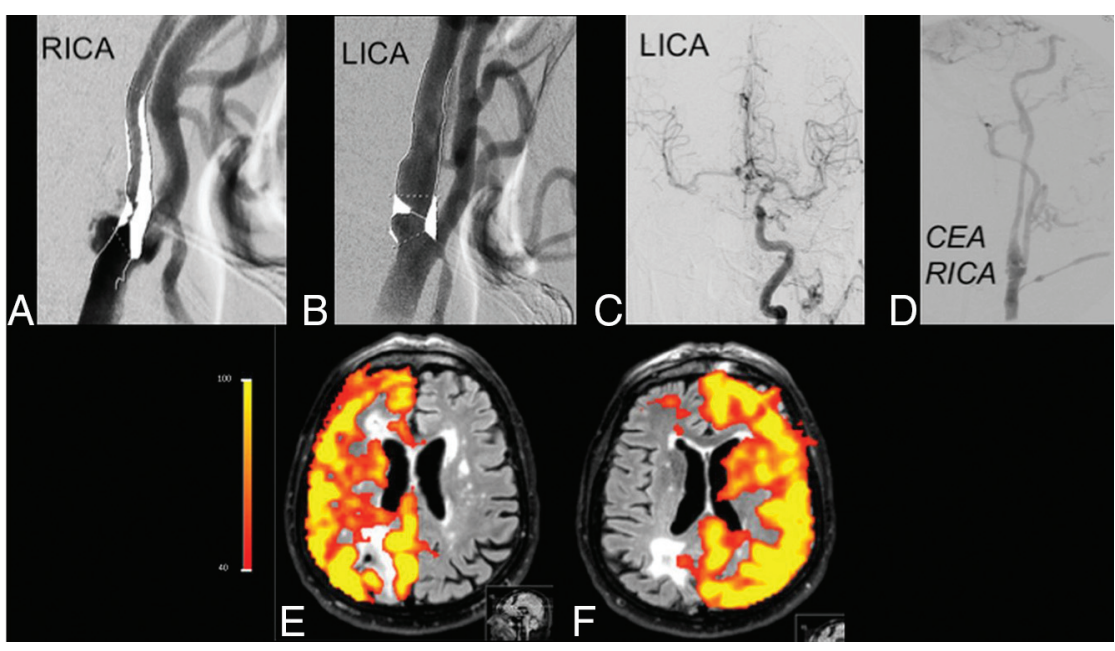

FIG 5. Initial CDSA shows high-grade ( $>70 \%)$ stenoses of both ICAs ( $A$ and $B)$, with flow on the right MCA and anterior cerebral artery from the left ICA (C). After carotid endarterectomy $(D)$, perfusion patterns of the right and left ICAs are normalized ( $E$ and $F$ ), demonstrating the utility of this noninvasive method for the follow-up of therapy success. RICA indicates right ICA; LICA, left ICA; CEA, carotid endarterectomy.

labeling spot can easily be performed by trained technicians and could also be applied in patients with complex vascular anatomy. In our experience, selective labeling was easy, even in the case of elongated vessels; we only labeled in the extracranial segment because there was more space for maneuvering. Qualitative perfusion territory maps for diagnostic assessment can, by correlation analysis, be produced in real-time at the scanner console, which is a major advantage compared with vessel-encoded pCASL, for which highly sophisticated postprocessing is required. ${ }^{19,25}$ In a subgroup of patients, the reliability of the method could be demonstrated via comparisons with DSC-based perfusion or DSA. In accordance with previous findings, ${ }^{11-13,19}$ we also demonstrated a high individual variability of perfusion patterns in our patient population with complex steno-occlusive disease of the extracranial brain-supplying arteries.

It had previously been demonstrated that even in a healthy population, a relevant variation in flow territories can be found, mainly due to anatomic variants of the circle of Willis. ${ }^{7,14,37}$ In chronic steno-occlusive disease, while hypotheses about perfusion territories may be postulated by imaging vascular anatomy, proof of hemodynamics is often required. ${ }^{11-13}$ DSA provides detailed information about macrovascular flow dynamics; however, it is an invasive procedure with an increased risk for cerebral embolisms. Especially in patients with steno-occlusive disease, plaque shear may result in cerebral embolism during the procedure. ${ }^{16}$ Superselective pCASL measurements are noninva- 
sive and do not require contrast agent application. This feature is important in patients with renal failure but is generally beneficial regarding recent reports on gadolinium deposition in the brain. ${ }^{38}$ In our patients, with, at most, mild cognitive impairments, the additional imaging time of approximately 10-15 minutes was well-tolerated; however, longer scan times may be a problem in severely debilitated patients with major stroke.

Superselective pCASL enables perfusion readout throughout the brain with accurate anatomic reference as an overlay. We found high agreement between superselective pCASL-based perfusion territories and DSA findings, in accordance with previous results. ${ }^{12}$ We also confirm previous findings that the circular focus labeling technique is especially useful for the exact labeling of tortuous cervical arteries, eliminating signal contamination by other arteries. $^{39}$

The visualization of the parenchymal FLAIR and DWI lesions in direct anatomic correlation with the regional perfusion territories enables determination of the etiology of infarct lesions with higher certainty because it allows detection of individual perfusion territories and watershed areas. According to current guidelines, patients with symptomatic carotid stenosis should be treated by revascularization (endarterectomy or stent placement) if the symptomatic stenosis is NASCET $>70 \%$ as documented by noninvasive imaging or NASCET $>50 \%$ as documented by conventional angiography. ${ }^{40-42}$ Therefore, precise imaging techniques are required for the correct identification of the symptomatic perfusion territories. Misidentification of perfusion territories ${ }^{43}$ may result in either overtreatment or a failure to treat a symptomatic stenosis. An example of such a complex case is shown in Fig 4, in which beyond chronic watershed infarcts between the stenotic ICA and the posterior perfusion territory, there was also evidence of an acute infarct, which could be allocated to the posterior perfusion territory by the use of ss-pCASL, demonstrating that the ICA stenosis was not responsible for the acute stroke.

In our patient cohort, we found a considerable percentage of patients with symmetric perfusion territories both in the group with unilateral high-grade ICA stenosis and in the group with bilateral ICA stenosis. It may be postulated that these patients, who do not have collateral recruitment, may be at risk for hemodynamic decompensation and future stroke events in the case of a progressing steno-occlusive disease because a new perfusion balance has not yet been established. While a decrease of cerebrovascular reactivity has been demonstrated in patients with an asymmetric perfusion pattern, ${ }^{44}$ no data are available for patients with unilateral stenosis and symmetric perfusion patterns. Identifying these patients may change their disease course by stringent follow-up and earlier intervention. Another potential application for regional perfusion imaging methods such as ss-pCASL may be the noninvasive follow-up of therapy success in this patient group, such as imaging normalization of perfusion territories after carotid endarterectomy or stent placement.

A limitation of this study is that only a small subset of patients underwent imaging with DSC (16/50) or DSA (12/50), preventing independent validation in most patients (22/50). A further limitation of the presented method may be the constant labeling duration and postlabeling delay, which were set to optimum values as previously described. ${ }^{45}$ These are somewhat shorter than the recommended values in the recently published consensus on ASL imaging. ${ }^{26}$ However, the effective delay time in this study varied between $1525 \mathrm{~ms}$ for the first (caudal) section to approximately $2000 \mathrm{~ms}$ for the last (cranial) section because a multisection EPI readout module was used. A short delay may cause overestimation of the CBF lesion; however, our results correlated well with the standard of DSC-PWI. The constant delay may influence readout quality in case of highly stenotic vessels, but perfusion territories being still easily discernible and distinguishable underlines the robustness of the method.

In the presented technique, a measurement of labeling efficiency was not performed for the sake of clinical feasibility. However, in applications concerned with evaluating pre- and poststenotic flow or vessels with slower flow such as the ECA, adjusting labeling duration and postlabeling delay may be crucial, even though the current recommendation suggests the use of single postlabeling-delay techniques for clinical applications. ${ }^{26}$ In our study, we did not label the ECA except for the cases with bilateral ICA occlusion; therefore, we did not assess ECA recruitment (reported to vary between $36 \%$ and $89 \%^{8}$ ). While ECA recruitment, along with leptomeningeal arteries, has an important role in acute collateral perfusion, ${ }^{46}$ retrograde ophthalmic perfusion has been reported to be regressive with time ${ }^{47}$; therefore, it may be less relevant in the compensated hemodynamic status of our patients with chronic stenoses.

Another potential limitation of the method includes its high sensitivity to motion; however, with proper patient instruction and positioning, this can be minimized. In addition, the planning scan can be repeated at any time to verify the correct positioning of the labeling focus, preventing missed labeling.

While the ss-pCASL sequence is currently only available for selected clinical users, which may limit a widespread application at this time, our data demonstrate its possible utility in the clinical setting.

\section{CONCLUSIONS}

We demonstrate that the clinical application of the superselective pCASL technique is feasible and that it facilitates the visualization of individual collateralization patterns in patients with complex extracranial steno-occlusive disease similar to cDSA. The regional perfusion imaging with ss-pcASL identifies individual perfusion patterns, which, in a relevant percentage of patients, cannot be predicted from the location of the extracranial vessel stenosis and the circle of Willis anatomy alone. It also improves the diagnosis of infarct genesis (embolic versus hemodynamic) and the source vessel. These findings have important implications for patient management. However, further technical refinements and multicenter studies are needed before a widespread clinical introduction of the method.

Disclosures: Michael Helle_UNRELATED: Employment: Philips Research. Mathias J.P. van Osch-UNRELATED: Payment for Lectures Including Service on Speakers Bureaus: Philips Healthcare. Claus Zimmer-UNRELATED: Board Membership: Philips Healthcare and Bayer Schering Pharma, Comments: scientific advisory boards*; Payment for Lectures Including Service on Speakers Bureaus: Bayer Schering Pharma and Philips Healthcare, Comments: speaker honoraria; Other: received research support and investigator fees for clinical studies from Biogen Idec, Quintiles, MSD Sharp \& Dome, Boehringer Ingelheim, InVentive Health Clinical UK Ltd, AdvanceCOR, BrainsGate, Pfizer, Bayer Schering Pharma, Novartis, Roche, Servier, Penumbra, WCT $\mathrm{GmbH}$, Syngis, SSS International Clinical Research, PPD Germany GmbH, Worldwide Clinical Trials, phenox, Covidien, Actelion, Medivation, Medtronic, Harrison Clinical 
Research, Concentric, Penumbra, pharmtrace, Reverse Medical Corp, Premier Research Germany Ltd, Surpass Medical Ltd, and GlaxoSmithKline. * *Money paid to the institution.

\section{REFERENCES}

1. Preibisch C, Richter V, Zimmer C. MR perfusion imaging. In: Hattingen E, Pilatus U, eds. Brain Tumor Imaging. Heidelberg: SpringerVerlag; 2014:75-98

2. Krabbe-Hartkamp MJ, van der Grond J, de Leeuw FE, et al. Circle of Willis: morphologic variation on three-dimensional time-of-flight MR angiograms. Radiology 1998;207:103-11 CrossRef Medline

3. Hendrikse J, van Raamt AF, van der Graaf Y, et al. Distribution of cerebral blood flow in the circle of Willis. Radiology 2005;235: 184-89 CrossRef Medline

4. Hendrikse J, Petersen ET, Chng SM, et al. Distribution of cerebral blood flow in the nucleus caudatus, nucleus lentiformis, and thalamus: a study of territorial arterial spin-labeling MR imaging. Radiology 2010;254:867-75 CrossRef Medline

5. van der Zwan A, Hillen B, Tulleken CA, et al. A quantitative investigation of the variability of the major cerebral arterial territories. Stroke 1993;24:1951-59 CrossRef Medline

6. van der Zwan A, Hillen B, Tulleken CA, et al. Variability of the territories of the major cerebral arteries. J Neurosurg 1992;77:927-40 CrossRef Medline

7. Hartkamp NS, Bokkers RP, van der Worp HB, et al. Distribution of cerebral blood flow in the caudate nucleus, lentiform nucleus and thalamus in patients with carotid artery stenosis. Eur Radiol 2011; 21:875-81 CrossRef Medline

8. van Laar PJ, van der Grond J, Bremmer JP, et al. Assessment of the contribution of the external carotid artery to brain perfusion in patients with internal carotid artery occlusion. Stroke 2008;39: 3003-08 CrossRef Medline

9. van Laar PJ, Hendrikse J, Klijn CJ, et al. Symptomatic carotid artery occlusion: flow territories of major brain-feeding arteries. Radiology 2007;242:526-34 CrossRef Medline

10. Van Laar PJ, Hendrikse J, Mali WP, et al. Altered flow territories after carotid stenting and carotid endarterectomy. J Vasc Surg 2007; 45:1155-61 CrossRef Medline

11. Kansagra AP, Wong EC. Quantitative assessment of mixed cerebral vascular territory supply with vessel encoded arterial spin labeling MRI. Stroke 2008;39:2980-85 CrossRef Medline

12. Chng SM, Petersen ET, Zimine I, et al. Territorial arterial spin labeling in the assessment of collateral circulation: comparison with digital subtraction angiography. Stroke 2008;39:3248-54 CrossRef Medline

13. Wu B, Wang X, Guo J, et al. Collateral circulation imaging: $M R$ perfusion territory arterial spin-labeling at 3T. AJNR Am J Neuroradiol 2008;29:1855-60 CrossRef Medline

14. van Laar PJ, Hendrikse J, Golay X, et al. In vivo flow territory mapping of major brain feeding arteries. Neuroimage 2006;29:136-44 CrossRef Medline

15. Liebeskind DS. Collateral circulation. Stroke 2003;34:2279-84 CrossRef Medline

16. Bendszus M, Koltzenburg M, Burger R, et al. Silent embolism in diagnostic cerebral angiography and neurointerventional procedures: a prospective study. Lancet 1999;354:1594-97 CrossRef Medline

17. Ter-Pogossian MM, Herscovitch P. Radioactive oxygen-15 in the study of cerebral blood flow, blood volume, and oxygen metabolism. Semin Nucl Med 1985;15:377-94 CrossRef Medline

18. van Laar PJ, van der Grond J, Hendrikse J. Brain perfusion territory imaging: methods and clinical applications of selective arterial spin-labeling MR imaging. Radiology 2008;246:354-64 CrossRef Medline

19. Bokkers RP, De Cocker LJ, van Osch MJ, et al. Selective arterial spin labeling: techniques and neurovascular applications. Top Magn Reson Imaging 2016;25:73-80 CrossRef Medline

20. Hartkamp NS, van Osch MJ, Kappelle J, et al. Arterial spin labeling magnetic resonance perfusion imaging in cerebral ischemia. Curr Opin Neurol 2014;27:42-53 CrossRef Medline

21. Davies NP, Jezzard P. Selective arterial spin labeling (SASL): perfusion territory mapping of selected feeding arteries tagged using two-dimensional radiofrequency pulses. Magn Reson Med 2003;49: 1133-42 CrossRef Medline

22. Helle M, Norris DG, Rüfer S, et al. Superselective pseudocontinuous arterial spin labeling. Magn Reson Med 2010;64:777-86 CrossRef Medline

23. Hartkamp NS, Helle M, Chappell MA, et al. Validation of planning free vessel-encoded pseudo-continuous arterial spin labeling MR imaging as territorial-ASL strategy by comparison to super-selective p-CASL MRI. Magn Reson Med 2014;71:2059-70 CrossRef Medline

24. Chappell MA, Okell TW, Jezzard P, et al. A general framework for the analysis of vessel encoded arterial spin labeling for vascular territory mapping. Magn Reson Med 2010;64:1529-39 CrossRef Medline

25. Wong EC. Vessel-encoded arterial spin-labeling using pseudocontinuous tagging. Magn Reson Med 2007;58:1086-91 CrossRef Medline

26. Alsop DC, Detre JA, Golay X, et al. Recommended implementation of arterial spin-labeled perfusion MRI for clinical applications: a consensus of the ISMRM perfusion study group and the European consortium for ASL in dementia. Magn Reson Med 2015;73:102-16 CrossRef Medline

27. Hendrikse J, van der Grond J, Lu H, et al. Flow territory mapping of the cerebral arteries with regional perfusion MRI. Stroke 2004;35: 882-87 CrossRef Medline

28. Werner R, Alfke K, Schaeffter T, et al. Brain perfusion territory imaging applying oblique-plane arterial spin labeling with a standard send/receive head coil. Magn Reson Med 2004;52:1443-47 CrossRef Medline

29. Möller HE, Mildner T, Preul C, et al. Assessment of collateral supply by two-coil continuous arterial spin labeling after coil occlusion of the internal carotid artery. AJNR Am J Neuroradiol 2007;28:1304-05 CrossRef Medline

30. Warmuth C, Rüping M, Förschler A, et al. Dynamic spin labeling angiography in extracranial carotid artery stenosis. AJNR Am J Neuroradiol 2005;26:1035-43 Medline

31. Hartkamp NS, Petersen ET, De Vis JB, et al. Mapping of cerebral perfusion territories using territorial arterial spin labeling: techniques and clinical application. NMR Biomed 2013;26:901-12 CrossRef Medline

32. Helle M, Rufer S, van Osch MJ, et al. Selective multivessel labeling approach for perfusion territory imaging in pseudo-continuous arterial spin labeling. Magn Reson Med 2012;68:214-19 CrossRef Medline

33. Osborn AG, Jacobs JM. Diagnostic Cerebral Angiography. Philadelphia: Lippincott-Raven; 1999

34. Tatu L, Moulin T, Bogousslavsky J, et al. Arterial territories of the human brain: cerebral hemispheres. Neurology 1998;50:1699-708 CrossRef Medline

35. Augustine JR. Human Neuroanatomy. London: Academic; 2007

36. Dice LR. Measures of the amount of ecologic association between species. Ecology 1945;26:297-302 CrossRef

37. Hartkamp MJ, van Der Grond J, van Everdingen KJ, et al. Circle of Willis collateral flow investigated by magnetic resonance angiography. Stroke 1999;30:2671-78 CrossRef Medline

38. Ramalho J, Ramalho M, Jay M, et al. Gadolinium toxicity and treatment. Magn Reson Imaging 2016;34:1394-98 CrossRef Medline

39. Helle M, Rüfer S, van Osch MJ, et al. Superselective arterial spin labeling applied for flow territory mapping in various cerebrovascular diseases. J Magn Reson Imaging 2013;38:496-503 CrossRef Medline

40. Rogers RK, Bishu K. Optimal treatment of extracranial carotid artery disease: carotid endarterectomy, carotid stenting, or optimal medical therapy. Curr Cardiol Rep 2015;17:84 CrossRef Medline

41. Brott TG, Halperin JL, Abbara S, et al. 2011 ASA/ACCF/AHA/ 
AANN/AANS/ACR/ASNR/CNS/SAIP/SCAI/SIR/SNIS/SVM/SVS guideline on the management of patients with extracranial carotid and vertebral artery disease: a report of the American College of Cardiology Foundation/American Heart Association Task Force on Practice Guidelines, and the American Stroke Association, American Association of Neuroscience Nurses, American Association of Neurological Surgeons, American College of Radiology, American Society of Neuroradiology, Congress of Neurological Surgeons, Society of Atherosclerosis Imaging and Prevention, Society for Cardiovascular Angiography and Interventions, Society of Interventional Radiology, Society of NeuroInterventional Surgery, Society for Vascular Medicine, and Society for Vascular Surgery. J Am Coll Cardiol 2011;57:e16-94 CrossRef Medline

42. Eckstein HH, Kühnl A, Dörfler A, et al; Multidisciplinary GermanAustrian guideline based on evidence and consensus. The diagnosis, treatment and follow-up of extracranial carotid stenosis. Dtsch Arztebl Int 2013;110:468-76 CrossRef Medline

43. Hartkamp NS, Hendrikse J, De Cocker LJ, et al. Misinterpretation of ischaemic infarct location in relationship to the cerebrovascular territories. J Neurol Neurosurg Psychiatry 2016;87:1084-90 CrossRef Medline

44. Bokkers RP, van Osch MJ, van der Worp HB, et al. Symptomatic carotid artery stenosis: impairment of cerebral autoregulation measured at the brain tissue level with arterial spin-labeling MR imaging. Radiology 2010;256:201-08 CrossRef Medline

45. Hendrikse J, van Osch MJ, Rutgers DR, et al. Internal carotid artery occlusion assessed at pulsed arterial spin-labeling perfusion MR imaging at multiple delay times. Radiology 2004;233:899-904 CrossRef Medline

46. Yamauchi H, Kudoh T, Sugimoto K, et al. Pattern of collaterals, type of infarcts, and haemodynamic impairment in carotid artery occlusion. J Neurol Neurosurg Psychiatry 2004;75:1697-701 CrossRef Medline

47. Rutgers DR, Klijn CJ, Kappelle LJ, et al, A longitudinal study of collateral flow patterns in the circle of Willis and the ophthalmic artery in patients with a symptomatic internal carotid artery occlusion. Stroke 2000;31:1913-20 CrossRef Medline 